\title{
X-ray absorption and magnetic circular dichroism of graphene/Ni(111)
}

\author{
Yu. S. Dedkov, ${ }^{1, a)}$ M. Sicot, ${ }^{2}$ and M. Fonin ${ }^{2}$ \\ ${ }^{1}$ Fritz-Haber Institut der Max-Planck Gesellschaft, 14195 Berlin, Germany \\ ${ }^{2}$ Fachbereich Physik, Universität Konstanz, 78457 Konstanz, Germany
}

\begin{abstract}
We present systematic investigations of the electronic and magnetic properties of the graphene/ $\mathrm{Ni}(111)$ system by means of $\mathrm{x}$-ray absorption spectroscopy (XAS) and magnetic circular dichroism (XMCD) at the Ni $L_{2,3}$ and $\mathrm{C} K$ absorption edges. The XAS C $1 s \rightarrow \pi^{*}, \sigma^{*}$ spectra show dramatic changes as the angle, $\alpha$, between the electrical vector of light and normal of the sample is varied reflecting the symmetry of the final state $(\sigma$ or $\pi)$. XMCD spectra reveal an induced magnetic moment of the carbon atoms in the graphene layer. Our experimental results are discussed in the light of previous results on the observation of induced magnetism in nonmagnetic materials. (C) 2010 American Institute of Physics.
\end{abstract}

Graphene, a single atom layer of hexagonally coordinated carbon $\left(s p^{2}\right.$-hybridized), has been found to exhibit exciting physical properties.' Among them a long electronic mean-free path and negligible spin-orbit coupling in graphene lead to large spin relaxation times, which render this material ideal for ballistic spin transport. Graphenebased spin electronic devices possess a tremendous potential for high-density nonvolatile memories, reconfigurable electronic devices and, possibly, solid-state quantum computing elements. ${ }^{2,3}$ One of the potential application of graphene is as a junction layer in spin-filtering devices. ${ }^{4}$ Such devices will require new materials to overcome some of the major problems currently hindering progress, such as low spin injection efficiency. ${ }^{5,6}$

Here we present a study of the electronic and magnetic properties of the graphene/ $\mathrm{Ni}(111)$ interface by means of photoelectron spectroscopy, angle-resolved x-ray absorption spectroscopy (XAS), and x-ray magnetic circular dichroism (XMCD) at the Ni $L_{2,3}$ and $\mathrm{C} K$ absorption edges. The XAS C $1 s \rightarrow \pi^{*}, \sigma^{*}$ spectra show pronounced changes as the angle, $\alpha$, between the electrical vector of light and plane of the sample is varied reflecting the symmetry of the final state $(\sigma$ or $\pi)$. XMCD reveals an induced magnetic moment of the carbon atoms in the graphene layer. Obtained experimental results are compared with recent observations of induced magnetism in nonmagnetic materials and magnetic moment of carbon atoms in the graphene layer is estimated.

The present experiments on the graphene/ $\mathrm{Ni}(111)$ system were performed at room temperature at the D1011 beamline of the MAX-Lab (Lund, Sweden). The base pressure in the experimental station is $5 \times 10^{-11}$ mbar. A well-ordered graphene/Ni(111) system was prepared according to the recipe described in Refs. 7-9. LEED and scanning tunnel microscope (STM) investigations of the graphene/Ni(111) system reveal a high quality of the system [Figs. 1(a) and 1(b)]. Photoemission spectra were obtained with SCIENTA200 energy analyzer with energy resolution of 100

${ }^{a)}$ Author to whom correspondence should be addressed. Electronic mail: dedkov@fhi-berlin.mpg.de.
meV. XAS and XMCD spectra were collected at both Ni $L_{2,3}$ and $C K$ absorption edges in partial electron yield (PEY) mode and total electron yield (TEY) mode with an energy resolution of $100 \mathrm{meV}$. Magnetic dichroism spectra were obtained with circularly polarized light (degree of polarization is $P=0.75$ ) at different angles $\alpha$ in the remanence magnetic state of the graphene/ $\mathrm{Ni}(111)$ system after applying of magnetic field of 500 Oe along the $\langle 1 \overline{1} 0\rangle$ easy magnetization axis of the $\mathrm{Ni}(111)$ thin film.

Figure 1(c) show the normal emission valence band spectra of single-crystalline graphite and graphene/Ni(111) which are in perfect agreement with the previously published data. ${ }^{8}$ The observed energy shift of $\sigma$ and $\pi$ valence band states to larger binding energy in case of the graphene layer on $\mathrm{Ni}(111)$ is different and can be explained by the different

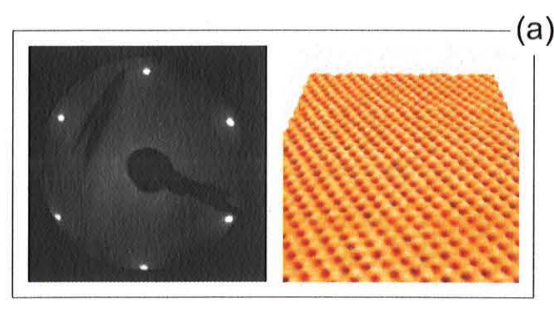

(a)

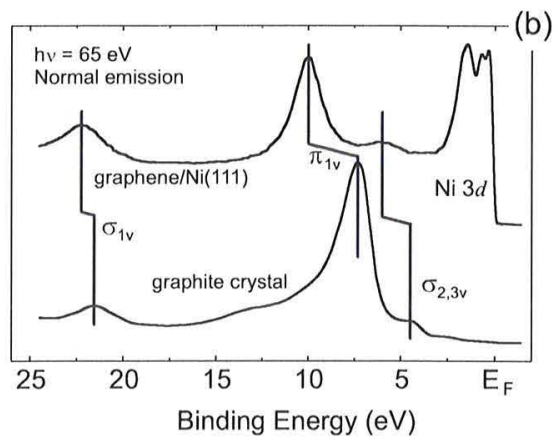

FIG. 1. (Color online) (a) A LEED pattern together with an STM image of the graphene layer on $\mathrm{Ni}(111)$. (c) Comparison of the normal emission valence band photoelectron spectra of the graphite crystal and graphene/ $\mathrm{Ni}(111)$ collected at $65 \mathrm{eV}$ of photon energy. 

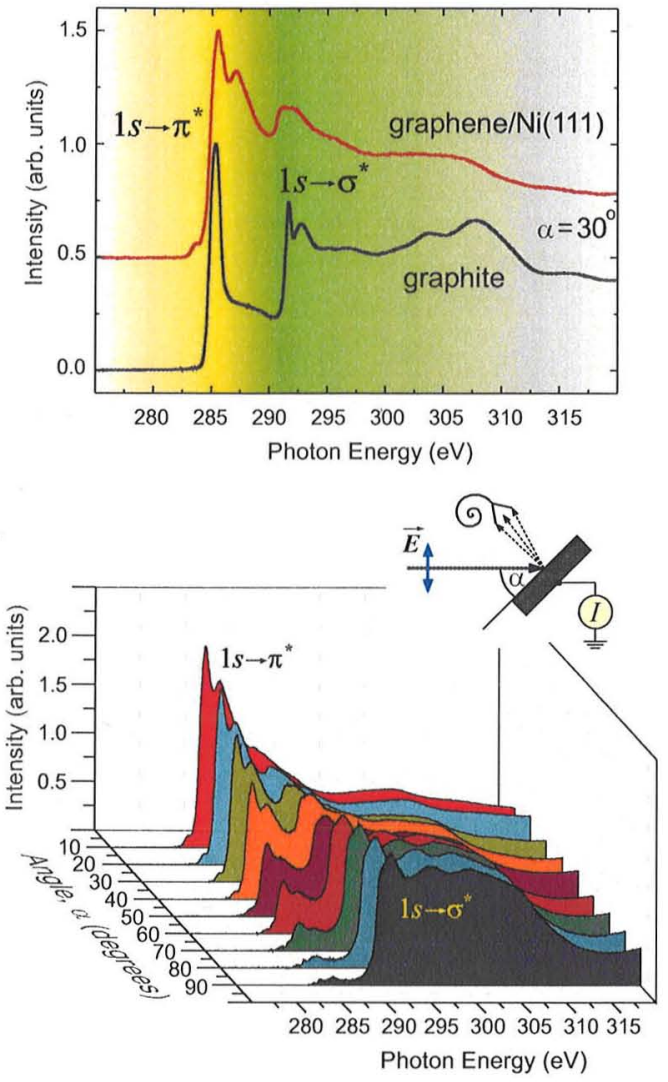

FIG. 2. (Color online) (Upper panel) XAS spectra of graphite and graphene/ $\mathrm{Ni}(111)$ measured at $\alpha=30^{\circ}$. (Lower panel) A series of the angle-dependent XAS spectra of the graphene/ $\mathrm{Ni}(111)$ system. See inset for the experimental geometry and notations.

strength of hybridization between these states and $\mathrm{Ni} 3 d$ valence band states. It is larger for the perpendicularly-to-theplane oriented $\pi$ states $(\approx 2.4 \mathrm{eV})$ compare to one for the in-plane oriented $\sigma$ states $(\approx 1 \mathrm{eV})$ of graphene. In this case due to the partial charge transfer from $\mathrm{Ni}$ atoms on carbon atoms of graphene, $\pi$ states become also spin-polarized, gaining a nonzero magnetic moment.

Figure 2 shows the XAS spectra of the graphene/Ni(111) system measured at the $\mathrm{C} K$ absorption edge as a function of the angle, $\alpha$, between the electrical vector of the light and the normal of the surface (see the inset of Fig. 2 for the geometry of the experiment). In these spectra two pronounced regions 283-289 $\mathrm{eV}$ and 289-315 eV are ascribed to $\mathrm{C} 1 s \rightarrow \pi^{*}$ and C $1 s \rightarrow \sigma^{*}$ transitions, respectively, of core electrons into $\pi^{*}$ and $\sigma^{*}$ orbitals. ${ }^{10}$ Comparison of the spectra collected for pure graphite and the graphene/Ni(111) system (Fig. 2, upper panel and Ref. 10) shows that the XAS C $1 s \rightarrow \pi^{*} \sigma^{*}$ spectrum is changed drastically indicating strong chemisorption in the later case. The same effects (but not so pronounced as in the present case) were recently observed for the graphene/ $\mathrm{Rh}(111)$ and graphene/Ru(0001) interfaces. ${ }^{11} \mathrm{~A}$ broadening of the $\pi^{*}$ and $\sigma^{*}$ resonances can also be taken as an evidence for the strong orbital hybridization and electron sharing at the graphene/ $\mathrm{Ni}$ interface indicating strong delocalization of the corresponding core-excited state. Comparison of the present results for graphene on $\mathrm{Ni}(111)$ with those obtained

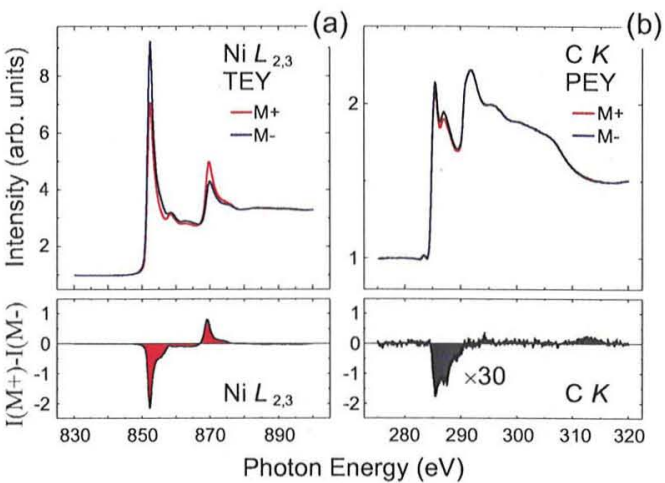

FIG. 3. (Color online) XMCD spectra of the graphene/Ni(111) system: absorption spectra measured for two opposite orientations of the magnetization are shown in the upper panels for the (a) $\mathrm{Ni} L_{2,3}$ and (b) $\mathrm{C} K$-edges. The corresponding differences are shown in the lower panels of the respective figures. In order to increase the measured magnetic contrast at the $1 s \rightarrow \pi^{*}$ absorption edge, these XMCD spectra were collected in the PEY mode with circularly polarized light at an angle of $\alpha=20^{\circ}$.

earlier for graphene/Rh and graphene/Ru indicates the existing of very strong covalent interfacial bonding between carbon atoms in the graphene layer and $\mathrm{Ni}$ atoms of the substrate.

The present XAS spectra of graphene/Ni(111) can be compared (to some extent) with the recently calculated $\mathrm{C} K$-edge electron energy loss spectra of this system. ${ }^{12}$ This analysis allows to assign the first peak in the $1 s \rightarrow \pi^{*}$ XAS spectra at $285.5 \mathrm{eV}$ of photon energy to the transition of the electron from $1 s$ core level on the interface state above the Fermi level (around $K$-point in the hexagonal Brillouin zone) originating from $\mathrm{C} p_{z}-\mathrm{Ni} 3 d$ hybridization and corresponding to antibonding between carbon atom C-top and interface $\mathrm{Ni}$ atom. The second peak in the spectrum at $287.1 \mathrm{eV}$ corresponds to the transition of the $1 s$ core electron on the interface state above the Fermi level (around $M$-point in the hexagonal Brillouin zone) originating from $\mathrm{C} p_{z}$ $-\mathrm{Ni} p_{x}, p_{y}, 3 d$ hybridization and corresponding to a bonding between the two carbon atoms, C-top and C- $f c c$, which involves the nickel interface atom. In case of the XAS C $1 s$ $\rightarrow \sigma^{*}$ theory also correctly describes the shape of the absorption spectra. ${ }^{12}$

Figure 3 shows XMCD spectra of the graphene/Ni(111) system collected at (a) $\mathrm{Ni} L_{2,3}$ and (b) $\mathrm{C} K$ absorption edges in TEY and PEY modes, respectively. The Ni $L_{2,3}$ XMCD spectrum is in the perfect agreement with previously published data. ${ }^{13}$ The bulk values of spin- and orbital-magnetic moments $\mu_{S}=0.69 \mu_{B}$ and $\mu_{L}=0.07 \mu_{B}$ of Ni calculated based on the sum-rules from the Ni $L_{2,3}$ TEY XAS spectra are in very good agreement with previously published experimental values. ${ }^{13,14}$

The most important finding within this experiment is, however, the observation of the relatively large XMCD contrast at the C $K$ absorption edge. The C $K$ XMCD spectrum reveals that the major magnetic response stems from transitions of the $1 s$ electron onto the $\pi^{*}$-states, while transitions onto the $\sigma^{*}$-states yield practically no magnetic signal indicating that only the C $2 p_{z}$ orbitals are polarized which hybridize with the Ni $3 d$ band. This is because the sharp struc- 
ture at the $1 s \rightarrow \pi^{*}$ absorption edge is originated from hybridized $\mathrm{C} p_{z}-\mathrm{Ni} 3 d$ and $\mathrm{C} p_{z}-\mathrm{Ni} p_{x}, p_{y}, 3 d$ states (see earlier discussion and Ref. 12).

At the $K$ edge, transitions occur from nonspin-orbit split $1 s$ initial states to $2 p$ final states. Thus, XMCD at the $K$ edge provides only the information on the orbital moment. ${ }^{15,16}$ Based on these observations we can conclude from the negative sign of the XMCD signal, that the orbital moment of the carbon atoms in the graphene layer is aligned parallel to both spin and orbital moments of the nickel substrate. The orientation of spin and orbital moments of both $\mathrm{Ni}$ and $\mathrm{C}$ is not straightforward evident from the experimental XMCD data.

Recently, two systems where induced magnetism on carbon atoms were under study. In the first one, $\mathrm{Fe} / \mathrm{C}$ multilayers, the clear magnetic signals of carbon were obtained by exploiting the standing-wave technique. ${ }^{17}$ In this system hysteresis loop of individually excited $\mathrm{C}$ atoms demonstrate ferromagnetism of carbon at room temperature with a moment of $0.05 \mu_{B}$ induced by adjacent Fe atoms. It was found that experimentally determined value is large than that $\left(0.02 \mu_{B}\right)$ estimated previously. ${ }^{18}$ Thus, magnetism in the $\mathrm{Fe} / \mathrm{C}$ multilayered system was related to the hybridization of the $\mathrm{Fe} 3 d$ orbitals and the $\mathrm{C} p_{z}$ orbitals which are normal to the graphene-type layered $s p^{2}$-coordination. In the second system the magnetic properties of carbon nanotubes on ferromagnetic Co substrate were studied. ${ }^{19}$ It was shown that carbon nanotubes can become magnetized when they are in contact with magnetic material. Spin-polarized charge transfer at the interface between a flat ferromagnetic metal substrate and a multiwalled carbon nanotube leads to a spin transfer of about $0.1 \mu_{B}$ per contact carbon atom.

In conclusion, the electronic structure and magnetic properties of the graphene/ $\mathrm{Ni}(111)$ system were studied by means of XAS and XMCD at the Ni $L_{2,3}$ and $\mathrm{C} K$ absorption edges. The XAS C $1 s \rightarrow \pi^{*}, \sigma^{*}$ spectra show pronounced changes upon a variation in the angle between the electrical vector of the light and the normal of the sample that reflects the symmetry of the final states $(\sigma$ or $\pi)$. XMCD experiments reveal an induced magnetic moment of the carbon atoms in the graphene layer. This magnetic moment originates from the strong hybridization between $\mathrm{C} \pi$ and $\mathrm{Ni} 3 d$ valence band states. Obtained experimental results are compared with the previous experimental observations of the induced magnetism in nonmagnetic materials.

This work was funded by the Deutsche Forschungsgemeinschaft, SFB 767, Project C5.

${ }^{1}$ A. Geim, Science 324, 1530 (2009).

${ }^{2}$ A. Rycerz, J. Tworzydlo, and C. W. J. Beenakker, Nat. Phys. 3, 172 (2007).

${ }^{3}$ B. Trauzettel, D. V. Bulaev, D. Loss, and G. Burkard, Nat. Phys. 3, 192 (2007).

${ }^{4}$ V. M. Karpan, G. Giovannetti, P. A. Khomyakov, M. Talanana, A. A. Starikov, M. Zwierzycki, J. van den Brink, G. Brocks, and P. J. Kelly, Phys. Rev. Lett. 99, 176602 (2007).

${ }^{5}$ G. Schmidt, D. Ferrand, L. W. Molenkamp, A. T. Filip, and B. J. van Wees, Phys. Rev. B 62, R4790 (2000).

${ }^{6}$ S. A. Wolf, D. D. Awschalom, R. A. Buhrman, J. M. Daughton, S. von Molnar, M. L. Roukes, A. Y. Chtchelkanova, and D. M. Treger, Science 294, 1488 (2001)

${ }^{7}$ Y. S. Dedkov, M. Fonin, and C. Laubschat, Appl. Phys. Lett. 92, 052506 (2008)

${ }^{8}$ Y. S. Dedkov, M. Fonin, U. Rüdiger, and C. Laubschat, Phys. Rev. Lett. 100, 107602 (2008)

${ }^{9}$ Y. S. Dedkov, M. Fonin, U. Rüdiger, and C. Laubschat, Appl. Phys. Lett. 93, 022509 (2008)

${ }^{10} \mathrm{P}$. Brühwiler, A. Maxwell, C. Puglia, A. Nilson, S. Anderson, and N. Martenson, Phys. Rev. Lett. 74, 614 (1995).

${ }^{11}$ A. B. Preobrajenski, M. L. Ng, A. S. Vinogradov, and N. Martensson, Phys. Rev. B 78, 073401 (2008).

${ }^{12}$ G. Bertoni, L. Calmels, A. Altibelli, and V. Serin, Phys. Rev. B 71, 075402 (2005)

${ }^{13}$ P. Srivastava, F. Wilhelm, A. Ney, M. Farle, H. Wende, N. Haack, G. Ceballos, and K. Baberschke, Phys. Rev. B 58, 5701 (1998).

${ }^{14}$ K. Baberschke, Appl. Phys. A: Mater. Sci. Process. 62, 417 (1996)

${ }^{15}$ B. Thole, P. Carra, F. Sette, and G. van der Laan, Phys. Rev. Lett. 68, 1943 (1992).

${ }^{16}$ P. Carra, B. Thole, M. Altarelli, and X. Wang, Phys. Rev. Lett. 70, 694 (1993).

${ }^{17}$ H. Mertins, S. Valencia, W. Gudat, P. Oppeneer, O. Zaharko, and H. Grimmer, Europhys. Lett. 66, 743 (2004).

${ }^{18}$ K. Murata, H. Ushijima, H. Ueda, and K. Kawaguchi, J. Chem. Soc., Chem. Commun. 1992, 567.

${ }^{19}$ O. Céspedes, M. S. Ferreira, S. Sanvito, M. Kociak, and J. M. D. Coey, J. Phys.: Condens. Matter 16, L155 (2004). 